\title{
ON $\theta$-GENERALIZED CLOSED SETS
}

\author{
JULIAN DONTCHEV and HARUO MAKI
}

(Received 14 October 1996 and in revised form 10 March 1998)

\begin{abstract}
The aim of this paper is to study the class of $\theta$-generalized closed sets, which is properly placed between the classes of generalized closed and $\theta$-closed sets. Furthermore, generalized $\Lambda$-sets [16] are extended to $\theta$-generalized $\Lambda$-sets and $R_{0^{-}}, T_{1 / 2^{-}}$and $T_{1^{-}}$-spaces are characterized. The relations with other notions directly or indirectly connected with generalized closed sets are investigated. The notion of TGO-connectedness is introduced.
\end{abstract}

Keywords and phrases. $\theta$-generalized closed, $\theta$-closure, $\Lambda$-set, TGO-connected.

1991 Mathematics Subject Classification. Primary 54H05, 54C08, 54D10; Secondary 54C10, 54D99.

1. Introduction. The first step of generalizing closed sets was done by Levine in 1970 [15]. He defined a set $A$ to be generalized closed if its closure belongs to every open superset of $A$ and introduced the notion of $T_{1 / 2}$-spaces, which is properly placed between $T_{0}$-spaces and $T_{1}$-spaces. Dunham [10] proved that a topological space is $T_{1 / 2}$ if and only if every singleton is open or closed. In [13], Khalimsky, Kopperman, and Meyer proved that the digital line is a typical example of a $T_{1 / 2}$-space.

Ever since, general topologists extended the study of generalized closed sets on the basis of generalized open sets: regular open, $\alpha$-open [20], semi-open [14], semipreopen [1], preopen [19], $\theta$-open [26], $\delta$-open [26], etc.

Extensive research on generalizing closedness was done in recent years as the notions of semi-generalized closed, generalized semi-closed, generalized $\alpha$-closed, $\alpha$ generalized closed, generalized semi-preclosed, regular generalized closed, $\gamma$-g-closed and $\left(\gamma, \gamma^{\prime}\right)$-g-closed sets were investigated [2, 3, 6, 7, 11, 18, 17, 22, 23, 24, 25].

Recently, in [8], Ganster and the first author of this paper defined $\delta$-generalized closed sets and introduced the notion of $T_{3 / 4}$-spaces, which is properly placed between $T_{1}$-spaces and $T_{1 / 2}$-spaces. They proved that the digital line is $T_{3 / 4}$.

The aim of this paper is to continue the study of generalized closed sets, this time via the $\theta$-closure operator defined in [26] and characterize $T_{1 / 2}$-spaces and $T_{1}$-spaces in terms of $\theta$-generalized closed sets. Via $\theta$-closure operator, we extend the class of generalized $\Lambda$-sets to the class of $\theta$-generalized $\Lambda$-sets and study some new characterizations of $R_{0}$-spaces and $T_{1}$-spaces.

2. Preliminaries concerning generalized closed sets. Throughout this paper, we consider spaces on which no separation axioms are assumed unless explicitly stated. The topology of a given space $X$ is denoted by $\tau$ and $(X, \tau)$ is replaced by $X$ if there is no chance for confusion. For $A \subseteq X$, the closure and the interior of $A$ in $X$ are denoted by $\mathrm{Cl}(A)$ and $\operatorname{Int}(A)$, respectively. Sometimes, when there is no chance for 
confusion, $\bar{A}$ stands for $\mathrm{Cl}(A)$. The $\theta$-interior [26] of a subset $A$ of $X$ is the union of all open sets of $X$ whose closures are contained in $A$, and is denoted by $\operatorname{Int}_{\theta}(A)$. The subset $A$ is called $\theta$-open [26] if $A=\operatorname{Int}_{\theta}(A)$. The complement of a $\theta$-open set is called $\theta$-closed. Alternatively, a set $A \subset(X, \tau)$ is called $\theta$-closed [26] if $A=\mathrm{Cl}_{\theta}(A)$, where $\mathrm{Cl}_{\theta}(A)=\{x \in X: \bar{U} \cap A \neq \varnothing, U \in \tau$ and $x \in U\}$. The family of all $\theta$-open sets forms a topology on $X$ and is denoted by $\tau_{\theta}$. We use the name CO-set for sets whose closure is open.

OBSERVATION 2.1. (i) If $A$ is preopen, then $\mathrm{Cl}_{\alpha}(A)=\mathrm{Cl}(A)=\mathrm{Cl}_{\theta}(A)$.

(ii) Every CO-set is preopen.

(iii) Every dense subset is a CO-set.

(iv) Every subset of a space $(X, \tau)$ is a CO-set if and only if $(X, \tau)$ is locally indiscrete.

Definition 1. A subset $A$ of a space $(X, \tau)$ is called

(1) a generalized closed set (=g-closed) [15] if $A \subseteq U$ and $U \in \tau$ implies that $\bar{A} \subseteq U$,

(2) a semi-generalized closed set (=sg-closed) [4] if $A \subseteq U$ and $U$ is semi-open implies that ${ }_{s} \mathrm{Cl}(A) \subseteq U$,

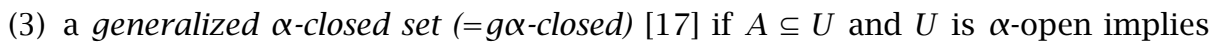
that $\mathrm{Cl}_{\alpha}(A) \subset U$,

(4) a generalized semi-closed set (= gs-closed) [2] if $A \subseteq U$ and $U \in T$ implies that ${ }_{\mathrm{s}} \mathrm{Cl}(A) \subseteq U$,

(5) an $\alpha$-generalized closed set ( $=\alpha$ g-closed) [18] if $A \subseteq U$ and $U \in T$ implies that $\mathrm{Cl}_{\alpha}(A) \subset U$

(6) a generalized semi-preclosed set (= gsp-closed) [7] if $A \subseteq U$ and $U \in \tau$ implies that ${ }_{\mathrm{sp}} \mathrm{Cl}(A) \subseteq U$,

(7) a regular generalized closed set (=r-g-closed) [23] if $A \subseteq U$ and $U$ is regular open implies that $\bar{A} \subseteq U$.

Definition 2. A topological space $(X, \tau)$ is called

(1) $R_{0}$-space [5] if the closures of every two different points are either disjoint or coincide,

(2) $R_{1}$-space [5] if every two different points, with distinct closures, have disjoint neighborhoods,

(3) $T_{1 / 2}$-space [15] if every g-closed set is closed, (= every singleton is open or closed [10]),

(4) kc-space [27] if every compact set is closed.

DEFINITION 3. Recall that a function $f:(X, \tau) \rightarrow(Y, \sigma)$ is called

(1) $g$-continuous [3] if $f^{-1}(V)$ is g-closed in $(X, \tau)$ for every closed set $V$ of $(Y, \sigma)$,

(2) semi-continuous [14] if $f^{-1}(V)$ is semi-open in $(X, \tau)$ for every open set $V$ of $(Y, \sigma)$,

(3) strongly $\theta$-continuous [21] if, for each $x \in X$ and each open set $V$ containing $f(x)$, there exists an open set $U$ containing $x$ such that $f(\bar{U}) \subseteq V$.

\section{Basic properties of $\theta$-generalized closed sets}

DeFINITION 4. A subset $A$ of a topological space $(X, \tau)$ is called $\theta$-generalized closed ( $=\theta$-g-closed) if $\mathrm{Cl}_{\theta}(A) \subseteq U$, whenever $A \subseteq U$ and $U$ is open in $(X, \tau)$. 
We denote the family of all $\theta$-generalized closed subsets of a space $(X, \tau)$ by $\operatorname{TGC}(X, \tau)$.

The next two results together with the examples following them show that the class of $\theta$-generalized closed sets is properly placed between the classes of g-closed and $\theta$-closed sets.

OBSERVATION 3.1. Every $\theta$-closed set is $\theta$-generalized closed.

EXAMPLE 3.2. Let $X=\{a, b, c\}$ and let $\tau=\{\varnothing,\{a, b\}, X\}$. Set $A=\{a, c\}$. Since the only open superset of $A$ is $X, A$ is clearly $\theta$-generalized closed. But it is easy to see that $A$ is not $\theta$-closed. In fact, it is not even semi-closed since its complement $\{b\}$ has empty interior.

OBSERVATION 3.3. Every $\theta$-generalized closed set is $g$-closed and hence $\alpha$ g-closed, gs-closed, and r-g-closed.

EXAMPLE 3.4. Let $X=\{a, b, c\}$ and let $\tau=\{\varnothing,\{a\},\{a, b\},\{a, c\}, X\}$. Set $A=\{c\}$. Clearly, $A$ is closed and hence g-closed. Next, set $U=\{a, c\}$. Note that $X=\mathrm{Cl}_{\theta}(A) \nsubseteq$ $U \in \tau$. Thus, $A$ is not $\theta$-generalized closed.

The following diagram is an enlargement of a Diagram from [7].

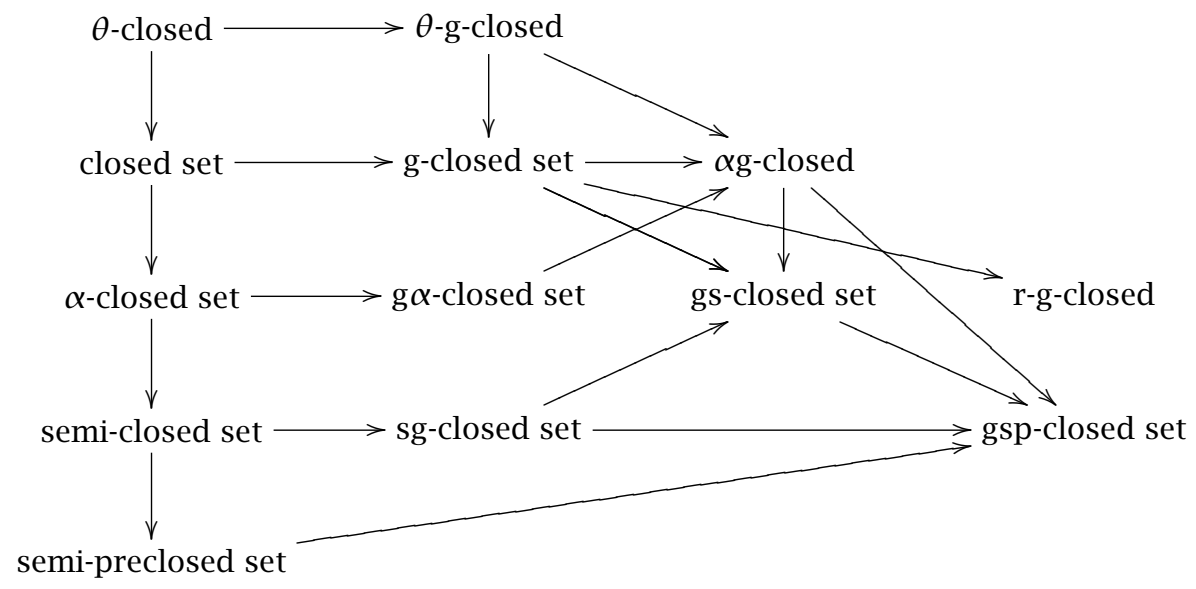

OBSERVATION 3.5. Let $(X, \tau)$ be a regular space (not necessarily even $T_{0}$ ). Then a subset $A$ of $X$ is $\theta$-generalized closed if and only if $A$ is generalized closed.

LEMмA 3.6 [12, Thm. 3.1(d), Thm. 3.6(d)]. For a space $(X, \tau)$, the following conditions are equivalent

(1) $X$ is an $R_{1}$-space;

(2) for each $x \in X, \mathrm{Cl}\{x\}=\mathrm{Cl}_{\theta}\{x\}$;

(3) for each compact set $A \subseteq X, \mathrm{Cl}(A)=\mathrm{Cl}_{\theta}(A)$.

Proposition 3.7. If $(X, \tau)$ is $R_{1}$, then a compact subset $K$ of $X$ is $g$-closed if and only if $K$ is $\theta$-g-closed.

Proposition 3.8. Let A be a preopen subset of a topological space $(X, \tau)$. Then the 
following conditions are equivalent

(1) $A$ is $\theta$-g-closed;

(2) $A$ is g-closed;

(3) $A$ is $\alpha g$-closed.

Proof. Follows easily from Observation 2.1(i) (note that a preopen g-closed set is a CO-set).

LEMMA 3.9. If $A$ and $B$ are subsets of a topological space $(X, \tau)$, then $\mathrm{Cl}_{\theta}(A \cup B)=$ $\mathrm{Cl}_{\theta}(A) \cup \mathrm{Cl}_{\theta}(B)$ and $\mathrm{Cl}_{\theta}(A \cap B) \subseteq \mathrm{Cl}_{\theta}(A) \cap \mathrm{Cl}_{\theta}(B)$.

Proposition 3.10. (i) A finite union of $\theta$-g-closed sets is always a $\theta$-g-closed set.

(ii) A countable union of $\theta$-g-closed sets need not be a $\theta$-g-closed set.

(iii) A finite intersection of $\theta-g$-closed sets may fail to be a $\theta$-g-closed set.

Proof. (i) Let $A, B \in \mathrm{TGC}(X)$. Let $U \in \tau$ such that $A \cup B \subseteq U$. By Lemma 3.9, $\mathrm{Cl}_{\theta}(A \cup$ $B)=\mathrm{Cl}_{\theta}(A) \cup \mathrm{Cl}_{\theta}(B) \subseteq U \cup U=U$ since $A$ and $B$ are $\theta$-g-closed. Hence, $A \cup B$ is $\theta$-gclosed.

(ii) Let $X$ be the real line with the usual topology. Since $X$ is regular, by Observation 3.5, every singleton in $X$ is $\theta$-g-closed. Set $A=\bigcup_{i=2}^{\infty}\{1 / i\}$. Clearly, $A$ is a countable union of $\theta$-generalized closed sets but $A$ is not $\theta$-generalized closed since $A \subseteq(0,1)$ and $0 \in \mathrm{Cl}_{\theta}(A)$.

(iii) Let $X=\{a, b, c, d, e\}$ and let $\tau=\{\varnothing,\{a, b\},\{c\},\{a, b, c\}, X\}$. Set $A=\{a, c, d\}$ and $B=\{b, c, e\}$. Clearly, $A$ and $B$ are $\theta$-generalized closed sets since $X$ is their only open superset. But $C=\{c\}=A \cap B$ is not $\theta$-generalized closed since $C \subseteq\{c\} \in \tau$ and $\mathrm{Cl}_{\theta}(C)=\{c, d, e\} \nsubseteq\{c\}$.

Proposition 3.11. The intersection of a $\theta$-generalized closed set and a $\theta$-closed set is always $\theta$-generalized closed.

Proof. Let $A$ be $\theta$-generalized closed and let $F$ be $\theta$-closed. Let $U$ be an open set such that $A \cap F \subseteq U$. Set $G=X \backslash F$. Then $A \subseteq U \cup G$. Since $G$ is $\theta$-open, $U \cup G$ is open and since $A$ is $\theta$-generalized closed, $\mathrm{Cl}_{\theta}(A) \subseteq U \cup G$. Now, by Lemma 3.9, $\mathrm{Cl}_{\theta}(A \cap F) \subseteq$ $\mathrm{Cl}_{\theta}(A) \cap \mathrm{Cl}_{\theta}(F)=\mathrm{Cl}_{\theta}(A) \cap F \subseteq(U \cup G) \cap F=(U \cap F) \cup(G \cap F)=(U \cap F) \cup \varnothing \subseteq U$.

Proposition 3.12. Let $B \subseteq H \subseteq(X, \tau)$ and $\left(\mathrm{Cl}_{\theta}\right)_{H}(B)$ denote the $\theta$-closure of $B$ in the subspace $(H, \tau \mid H)$. Then

(i) $\left(\mathrm{Cl}_{\theta}\right)_{H}(B) \subseteq \mathrm{Cl}_{\theta}(B) \cap H$ holds.

(ii) If $H$ is open in $(X, \tau)$, then $\left(\mathrm{Cl}_{\theta}\right)_{H}(B) \supset \mathrm{Cl}_{\theta}(B) \cap H$ holds.

THEOREM 3.13. Let $B \subseteq H \subseteq(X, \tau)$.

(i) If $B$ is $\theta$-g-closed relative to $H$ (i.e., $B \in \mathrm{TGC}(H, \tau \mid H)), H \in \mathrm{TGC}(X)$, and $H \in \tau$, then $B \in \operatorname{TGC}(X)$.

(ii) If $B$ is $\theta$-g-closed in $(X, \tau)$, then $B$ is $\theta$-g-closed relative to $H$ (i.e., $B \in \operatorname{TGC}(H, \tau \mid$ $H)$ ).

Proof. (i) Let $B \subseteq U$, where $U \in \tau$. Then $B \subseteq H \cap U$ and, moreover, $\left(C l_{\theta}\right)_{H}(B) \subseteq$ $H \cap U$ due to assumption. By Proposition 3.12(ii), $H \cap \mathrm{Cl}_{\theta}(B) \subseteq H \cap U \subseteq U$. Using the last inclusion, it follows that $H \subseteq H \cup\left(X \backslash \mathrm{Cl}_{\theta}(B)\right)=\left(H \cap \mathrm{Cl}_{\theta}(B)\right) \cup\left(X \backslash \mathrm{Cl}_{\theta}(B)\right) \subseteq U \cup$ $\left(X \backslash \mathrm{Cl}_{\theta}(B)\right)$. Since $\mathrm{Cl}_{\theta}(B)$ is a closed set, $U \cup\left(X \backslash \mathrm{Cl}_{\theta}(B)\right)$ is open and thus since $H \in$ $\operatorname{TGC}(X), \mathrm{Cl}_{\theta}(H) \subseteq U \cup\left(X \backslash \mathrm{Cl}_{\theta}(B)\right)$. Now, $\mathrm{Cl}_{\theta}(B) \subseteq \mathrm{Cl}_{\theta}(H) \subseteq U \cup\left(X \backslash \mathrm{Cl}_{\theta}(B)\right)$. From the 
last inclusion, it follows that $\mathrm{Cl}_{\theta}(B) \subseteq U$ or, equivalently, $B \in \operatorname{TGC}(X)$.

(ii) Let $V$ be an open set of $(H, \tau \mid H)$ such that $B \subset V$. Then there exists an open set $G \in \tau$ such that $G \cap H=V$. Since $B \subseteq G \cap H \subseteq G$ and $B \in \operatorname{TGC}(X), \mathrm{Cl}_{\theta}(B) \subseteq G$. By Proposition 3.12(i), $\left(\mathrm{Cl}_{\theta}\right)_{H}(B) \subseteq \mathrm{Cl}_{\theta}(B) \cap H \subseteq G \cap H \subseteq V$. Therefore, $B$ is $\theta$-g-closed relative to $H$.

EXAMPLE 3.14. Let $X=\{a, b, c, d\}$ and $\tau=\{\varnothing,\{a\},\{a, b\},\{a, c, d\}, X\}$. Then $\{\varnothing, X\}$ is the set of all $\theta$-closed sets of $(X, \tau)$ and $\operatorname{TGC}(X, \tau)=\{\varnothing,\{b, c\},\{b, d\},\{b, c, d\}$, $\{a, b, d\},\{a, b, c\}, X\}$. Let $H=\{b, c, d\}$ be a set of $X$. Then, $\tau \mid H=\{\varnothing,\{b\},\{c, d\}, H\}$. Note that $\{\varnothing,\{b\},\{c, d\}, H\}$ is the set of all $\theta$-closed sets of $(H, \tau \mid H)$ and $\operatorname{TGC}(H, \tau \mid$ $H)=\mathscr{P}(H)$. The subset $\{\boldsymbol{b}\}$ of $H$ is $\theta$-g-closed relative to $H$ and $H$ is not open (i.e., $\{b\} \in$ $\operatorname{TGC}(H, \boldsymbol{\tau} \mid H), H \notin \boldsymbol{\tau})$ and $H \in \operatorname{TGC}(X, \boldsymbol{\tau})$. However, $\{\boldsymbol{b}\} \notin \operatorname{TGC}(X, \boldsymbol{\tau})$.

EXAMPLE 3.15. Let $(X, \tau)$ be the space in the example above. Set $H=\{a, c, d\}$. Clearly, $H$ is open in $(X, \tau)$ and $H$ is not $\theta$-generalized closed in $(X, \tau)$. But $B=\{a, c\}$ is $\theta$-generalized closed relative to H. However, B is not $\theta$-generalized closed in $(X, \tau)$.

\section{Characterizations of $T_{1 / 2}$-spaces, $T_{1}$-spaces and $R_{0}$-spaces}

THEOREM 4.1. A space $(X, \tau)$ is a $T_{1 / 2}$-space if and only if every $\theta$-generalized closed set is closed.

PROOF.

NeCESSITY. Let $A \subseteq X$ be $\theta$-generalized closed. By Observation 3.3, $A$ is g-closed. Since $X$ is a $T_{1 / 2}$-space, $A$ is closed.

SuFFICIENCY. Let $x \in X$. If $\{x\}$ is not closed, then $B=X \backslash\{x\}$ is not open and thus the only superset of $B$ is $X$. Trivially, $B$ is $\theta$-generalized closed. By (2), $B$ is closed or, equivalently, $\{x\}$ is open. Thus, every singleton in $X$ is open or closed. Hence, in the notion of [6, Thm. 6.2(i)], $X$ is a $T_{1 / 2}$-space.

LEMMA 4.2. Let $A \subseteq(X, \tau)$ be $\theta$-generalized closed. Then $\mathrm{Cl}_{\theta}(A) \backslash A$ does not contain a nonempty closed set.

THEOREM 4.3. A space $(X, \tau)$ is a $T_{1}$-space if and only if every $\theta$-generalized closed set is $\theta$-closed.

\section{PROOF.}

NeCESSITY. Let $A \subseteq X$ be $\theta$-generalized closed and let $x \in \mathrm{Cl}_{\theta}(A)$. Since $X$ is $T_{1}$, $\{x\}$ is closed and thus by Lemma 4.2, $x \notin \mathrm{Cl}_{\theta}(A) \backslash A$. Since $x \in \mathrm{Cl}_{\theta}(A)$, then $x \in A$. This shows that $\mathrm{Cl}_{\theta}(A) \subseteq A$ or, equivalently, that $A$ is $\theta$-closed.

SUFFICIENCY. Let $x \in X$. Assume that $\{x\}$ is not closed. Then $B=X \backslash\{x\}$ is not open and, trivially, $B$ is $\theta$-generalized closed since the only open superset of $B$ is $X$ itself. By (2), $B$ is $\theta$-closed and thus $\{x\}$ is $\theta$-open. Since a singleton is $\theta$-open if and only if it is clopen, $\{x\}$ is clopen.

The notion of a $\Lambda$-set and a generalized $\Lambda$-set in a topological space was introduced in [16]. By definition, a subset $A$ of a topological space $(X, \tau)$ is called a $\Lambda$-set [16] if $A=A^{\Lambda}$, where $A^{\Lambda}=\cap\{U: U \supset A, U \in \tau\}$. Recall that $A$ is called a generalized $\Lambda$-set [16] if $A^{\Lambda} \subseteq F$, whenever $A \subseteq F$ and $F$ is $\tau$-closed. 
DEFinition 5. (i) For a subset $A$ of $(X, \tau)$, we define $A_{\theta}^{\Lambda}$ as follows

$$
A_{\theta}^{\Lambda}=\left\{x \in X: \operatorname{Cl}_{\theta}\{x\} \cap A \neq \varnothing\right\} .
$$

In [12], $A_{\theta}^{\Lambda}$ is denoted by $\operatorname{ker}_{\theta} A$.

(ii) A subset $A$ of $(X, \tau)$ is called $\theta$-generalized $\Lambda$-set $(=\theta$-g- $\Lambda$-set $)$ if $A_{\theta}^{\Lambda} \subseteq F$, whenever $A \subseteq F$ and $F$ is closed in $(X, \tau)$.

OBSERVATION 4.4. (i) Every $G_{\delta}$-set is a $\Lambda$-set.

(ii) $\left[12\right.$, Lem. 3.5(a)]. For any set $A \subseteq X, A \subseteq A^{\Lambda} \subseteq A_{\theta}^{\Lambda} \subseteq \mathrm{Cl}_{\theta}(A)$.

(iii) Every $\theta$-closed set is a $\Lambda$-set.

(iv) Every g-closed $\Lambda$-set is closed.

(v) Every $\theta$-generalized $\Lambda$-set is a generalized $\Lambda$-set.

REMARK 4.5. (i) A $\Lambda$-set need not be $\theta$-closed. Any singleton of an infinite space with the cofinite topology is a $\Lambda$-set (since the space is $T_{1}$ ) but none of the singletons is $\theta$-closed.

(ii) A closed set need not be a $\Lambda$-set. In the Sierpinski space $(X=\{a, b\}, \tau=\{\varnothing,\{a\}$, $X\}$ ), the set $B=\{b\}$ is closed but $B$ is not a $\Lambda$-set. However, in [16, Prop. 3.8], it was shown that in a topological space $(X, \tau)$, every subset of $X$ is a generalized $\Lambda$-set if and only if every closed set is a $\Lambda$-set.

(iii) A generalized $\Lambda$-set need not be $\theta$-generalized $\Lambda$-set. In an infinite cofinite space $X$, as mentioned in Remark 4.5, every singleton is a $\Lambda$-set and, hence, a generalized $\Lambda$-set but none of the singletons is a $\theta$-generalized $\Lambda$-set since the $\theta$-closure of every singleton is $X$.

In [16], it was proved that in $T_{1}$-spaces, every set is a $\Lambda$-set. Note that the converse is also true.

Proposition 4.6. (i) A topological space $(X, \tau)$ is a $T_{1}$-space if and only if every subset of $X$ is a $\Lambda$-set.

(ii) A topological space $(X, \tau)$ is an $R_{0}$-space if and only if every singleton of $X$ is a generalized $\Lambda$-set.

Proof. (i) Obvious.

(ii) In [9], Dube showed that a space is $R_{0}$ if and only if, for each closed set $A$, $A=A^{\Lambda}$. Thus, if $X$ is $R_{0}$, then for each singleton $\{x\}$ and each closed set $F$ containing $x$, we have $\{x\} \subseteq\{x\}^{\Lambda} \subseteq F^{\Lambda}=F$. So, $\{x\}$ is a generalized $\Lambda$-set. For the reverse assume that $F \subseteq X$ is closed. For each $x \in F$, by assumption, $\{x\}^{\Lambda} \subseteq F$. Thus, $F^{\Lambda}=\cup_{x \in F}\{x\}^{\Lambda} \subseteq$ $F$ according to [16, condition (2.5)]. This shows that $F=F^{\Lambda}$.

OBSERVATION 4.7. (i) A subset $A$ of an $R_{1}$-space $X$ is generalized $\Lambda$-set if and only if $A$ is $\theta$-generalized $\Lambda$-set.

(ii) In Hausdorff spaces, every subset is a $\theta$-generalized $\Lambda$-set.

(iii) A topological space $X$ is Hausdorff if and only if $X$ is a kc-space and every closed set of $X$ is a $\theta$-generalized $\Lambda$-set.

\section{5. $\theta$-g-continuous and $\theta$-g-irresolute functions}

DEFINITION 6. A function $f:(X, \tau) \rightarrow(Y, \sigma)$ is called 
(1) $\theta$-g-continuous if $f^{-1}(V)$ is $\theta$-g-closed in $(X, \tau)$ for every closed set $V$ of $(Y, \sigma)$,

(2) $\theta$-g-irresolute if $f^{-1}(V)$ is $\theta$-g-closed in $(X, \tau)$ for every $\theta$-g-closed set $V$ of $(Y, \sigma)$.

OBSERVATION 5.1. If $f:(X, \tau) \rightarrow(Y, \sigma)$ is strongly $\theta$-continuous, then $f$ is $\theta$-gcontinuous.

EXAMPLE 5.2. Let $(X, \boldsymbol{\tau})$ be the space in Example 3.2. Let $\sigma=\{\varnothing,\{b\}, X\}$. Let $f$ : $(X, \tau) \rightarrow(X, \sigma)$ be the identity function. Clearly, in the notion of Example 3.2, $f$ is $\theta$-g-continuous but $f$ is not strongly $\theta$-continuous, not even semi-continuous.

OBSERVATION 5.3. Let $f:(X, \tau) \rightarrow(Y, \sigma)$ be $\theta$-g-continuous. Then $f$ is $g$-continuous but not conversely.

EXAMPLE 5.4. Let $(X, \tau)$ be the space in Example 3.4. Let $\sigma=\{\varnothing,\{a, b\}, X\}$. Let $f:(X, \tau) \rightarrow(X, \sigma)$ be the identity function. Clearly, $f$ is continuous and hence $g$ continuous but as shown in Example 3.4, $A=\{c\} \notin \operatorname{TGC}(X, \tau)$ and hence $f$ is not $\theta$-g-continuous.

Example 5.2 and Example 5.4 also show that continuity and $\theta$-g-continuity are independent concepts. Thus, we have the following implications and none of them is reversible.

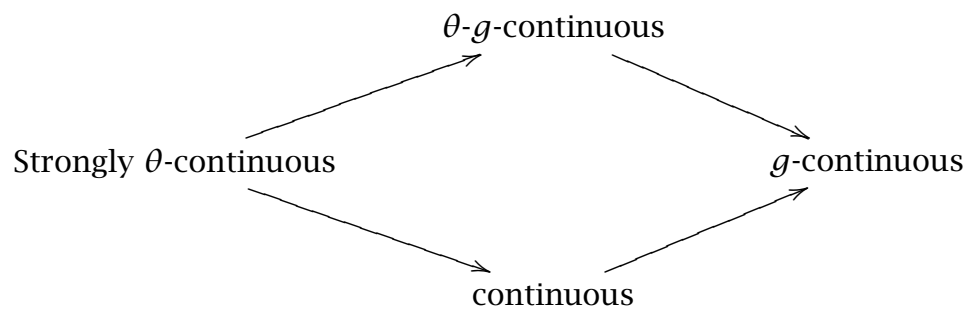

EXAMPLE 5.5. Let $f$ be the function in Example 5.2. Let $v=\{\varnothing,\{c\}, X\}$. Let $g:(X, \sigma) \rightarrow(X, v)$ be the identity function. It is easily observed that $g$ is also $\theta$ generalized continuous. But the composition function $g \circ f:(X, \tau) \rightarrow(X, v)$ is not $\theta$-generalized continuous since $\{a, b\} \notin \operatorname{TGC}(X, \tau)$.

THEOREM 5.6. If $f:(X, \tau) \rightarrow(Y, \sigma)$ is bijective, open and $\theta$-generalized continuous, then $f$ is $\theta$-g-irresolute.

Proof. Let $V \in \operatorname{TGC}(Y)$ and let $f^{-1}(V) \subseteq O$, where $O \in \tau$. Clearly, $V \subseteq f(O)$. Since $f(O) \in \sigma$ and since $V \in \operatorname{TGC}(Y), \mathrm{Cl}_{\theta}(V) \subseteq f(O)$ and thus $f^{-1}\left(\mathrm{Cl}_{\theta}(V)\right) \subset O$. Since $f$ is $\theta$-generalized continuous and since $\mathrm{Cl}_{\theta}(V)$ is closed in $Y, \mathrm{Cl}_{\theta}\left(f^{-1}\left(\mathrm{Cl}_{\theta}(V)\right)\right) \subseteq O$ and hence $\mathrm{Cl}_{\theta}\left(f^{-1}(V)\right) \subseteq O$. Therefore, $f^{-1}(V) \in \operatorname{TGC}(X)$. Hence, $f$ is $\theta$-g-irresolute.

DEFINITION 7. A function $f:(X, \tau) \rightarrow(Y, \sigma)$ is called $\theta$-generalized closed if, for every closed set $F$ of $(X, \tau), f(F)$ is $\theta$-g-closed in $(Y, \sigma)$.

THEOREM 5.7. (i) Let $f:(X, \tau) \rightarrow(Y, \sigma)$ be continuous and $\theta$-generalized closed. Then, for a $\theta$-g-closed set $A$ of $X, f(A)$ is $\theta$-g-closed in $Y$. 
(ii) Let $f:(X, \tau) \rightarrow(Y, \sigma)$ be strongly $\theta$-continuous and closed. Then, $f$ is $\theta$-girresolute.

Proof. (i) Left to the reader.

(ii) Let $B$ be a $\theta$-g-closed set of $(Y, \sigma)$ and let $U \in \tau$ such that $f^{-1}(B) \subseteq U$. Put $H=\mathrm{Cl}_{\theta}\left(f^{-1}(B)\right) \cap(X \backslash U)$. A map $f:(X, \tau) \rightarrow(Y, \sigma)$ is strongly $\theta$-continuous if and only if $f:(X, \tau) \rightarrow(Y, \sigma)$ is $(\gamma$, id $)$-continuous in the sense of Ogata [22, Def. 4.12], where $\gamma: \tau \rightarrow \mathscr{P}(X)$ is the closure operation and id $: \sigma \rightarrow \mathscr{P}(Y)$ is the identity operation. Using [22, Prop. 4.13(i)] and the fact that $\mathrm{Cl}_{\gamma}(E)=\mathrm{Cl}_{\theta}(E)$ and $\mathrm{Cl}_{\mathrm{id}}(E)=$ $\mathrm{Cl}(E)$ for the closure operation $\gamma$, the identity operation id and the subset $E$, we get $f(H) \subseteq f\left(\mathrm{Cl}_{\theta}\left(f^{-1}(B)\right)\right) \cap f(X \backslash B) \subseteq \mathrm{Cl}\left(f\left(f^{-1}(B)\right)\right) \cap(X \backslash B) \subseteq \mathrm{Cl}(B) \backslash B \subset \mathrm{Cl}_{\theta}(B) \backslash B$. By Lemma 4.2, $f(H)=\varnothing$ since $f(H)$ is closed. We have $H=\varnothing$ and hence $\mathrm{Cl}_{\theta}\left(f^{-1}(B)\right) \subseteq$ $U$. Therefore, $f^{-1}(B) \in \mathrm{TGC}(X, \tau)$.

COROLLARY 5.8. (i) Under the same assumptions of Theorem 5.6, if $(X, \tau)$ is $T_{1 / 2}$, then $(Y, \sigma)$ is $T_{1 / 2}$.

(ii) Under the same assumptions of Theorem 5.7(ii), if $(X, \tau)$ is $T_{1 / 2}$ and $f:(X, \tau) \rightarrow$ $(Y, \sigma)$ is surjective, then $(Y, \sigma)$ is $T_{1 / 2}$.

Proposition 5.9. Let $f:(X, \tau) \rightarrow(Y, \sigma)$ be a $\theta$-generalized continuous function and let $H$ be a $\theta$-closed subset of $X$. Then the restriction $f \mid H:(H, \tau \mid H) \rightarrow(Y, \sigma)$ is $\theta$-generalized continuous.

Proof. Let $F$ be a closed subset of $(Y, \sigma)$. By Proposition 3.11, $H_{1}=f^{-1}(F) \cap H$ is $\theta$-generalized closed in $(X, \tau)$. Then, by Theorem 3.13(ii), $H_{1}$ is $\theta$-g-closed in $(H, \tau \mid H)$. Since $(f \mid H)^{-1}(F)=H_{1}, f \mid H$ is $\theta$-g-continuous.

Next, we offer the following "Pasting Lemma" for $\theta$-g-continuous functions.

Proposition 5.10. Let $(X, \tau)$ be a topological space such that $X=A \cup B$, where both $A, B \in \mathrm{TGC}(X)$ and $A, B \in \tau$. Let $f:(A, \tau \mid A) \rightarrow(Y, \sigma)$ and $g:(B, \tau \mid B) \rightarrow(Y, \sigma)$ be $\theta$-generalized continuous functions such that $f(x)=g(x)$ for every $x \in A \cap B$. Then the combination $\alpha:(X, \tau) \rightarrow(Y, \sigma)$ is $\theta$-generalized continuous, where $\alpha(x)=f(x)$ for any $x \in A$ and $\alpha(y)=g(y)$ for any $y \in B$.

DEFINITION 8. A subset $A$ of $(X, \tau)$ is called $\theta$-generalized open ( $=\theta$-g-open) if its complement $X \backslash A$ is $\theta$-generalized closed in $(X, \tau)$.

THEOREM 5.11. (i) $A$ subset $A$ of $(X, \tau)$ is $\theta$-g-open if and only if $F \subseteq \operatorname{Int}_{\theta}(A)$, whenever $F \subset A$ and $F$ is closed in $(X, \tau)$.

(ii) If $A$ is $\theta$-g-open in $(X, \tau)$ and $B$ is $\theta$-g-open in $(Y, \sigma)$, then $A \times B$ is $\theta$-g-open in the product space $(X \times Y, \tau \times \sigma)$.

Proof. (i) Obvious.

(ii) Let $F$ be a closed subset of $(X \times Y, \tau \times \sigma)$ such that $F \subseteq A \times B$. For each $(x, y) \in$ $F, \mathrm{Cl}(\{x\}) \times \mathrm{Cl}(\{y\}) \subseteq \mathrm{Cl}(F)=F \subseteq A \times B$. Then the two closed sets $\mathrm{Cl}(\{x\})$ and $\mathrm{Cl}(\{y\})$ are contained in $A$ and $B$, respectively. By assumption, $\mathrm{Cl}(\{x\}) \subseteq \operatorname{Int}_{\theta}(A)$ and $\mathrm{Cl}(\{y\}) \subseteq \operatorname{Int}_{\theta}(B)$ hold. This implies that, for each $(x, y) \in F,(x, y) \in \operatorname{Int}_{\theta}(A) \times$ $\operatorname{Int}_{\theta}(B) \subseteq \operatorname{Int}_{\theta}(A \times B)$ and hence $F \subset \operatorname{Int}_{\theta}(A \times B)$. By (i) it is clear that $A \times B$ is $\theta$-g-open. 
Proposition 5.12. The projection $p:(X \times Y, \tau \times \sigma) \rightarrow(X, \tau)$ is a $\theta$-g-irresolute map.

Proof. By definition and Theorem 5.11(ii), for a $\theta$-generalized closed set $F$ of $(X, \tau), p^{-1}(x \backslash F)=(X \backslash F) \times Y$ is $\theta$-g-open in $(X \times Y, \tau \times \sigma)$. Therefore, $P^{-1}(F)=F \times Y=$ $X \times Y \backslash\left(p^{-1}(X \backslash F)\right)$ is $\theta$-generalized closed.

6. TGO-connected spaces. In 1991, Balachandran et al. [3] introduced a stronger form of connectedness called GO-connectedness. A set is called g-open [15] if its complement is g-closed.

DefinITION 9. (cf. [15]). A topological space $X$ is called TGO-connected (respectively, GO-connected [15]) if $X$ cannot be written as a disjoint union of two nonempty $\theta$-g-open (respectively, g-open) sets. A subset of $X$ is called TGO-connected if it is connected as a subspace.

Clearly, every TGO-connected space is connected. The space in [3, Ex. 11] shows that there are connected spaces which are not TGO-connected. Since every $\theta$-generalized closed set is g-closed, every GO-connected space is TGO-connected. Thus, we have the following implications and none of them is reversible.

$$
\text { GO-connected } \Longrightarrow \text { TGO-connected } \Longrightarrow \text { Connected }
$$

EXAMPLE 6.1. Let $X=\{a, b, c, d\}$ and let $\tau=\{\varnothing,\{a\},\{a, b\},\{a, c, d\}, X\}$. Since $\{c\}$ is both g-closed and g-open, $X$ is not GO-connected. Note that $\operatorname{TGC}(X)=\{\varnothing,\{b, c\}$, $\{b, d\},\{a, b, c\},\{a, b, d\},\{b, c, d\}, X\}$. Hence, $X$ is TGO-connected.

ObSERVATION 6.2. (i) [3, Prop. 10]. For a topological space $(X, \tau)$, the following conditions are equivalent.

(1) $X$ is TGO-connected;

(2) the only subsets of $X$, which are both $\theta$-g-open and $\theta$-g-closed, are $\varnothing$ and $X$;

(3) each $\theta$-generalized continuous function of $X$ into a discrete space $Y$, with at least two points, is constant.

(ii) [3, Prop. 12]. If $(X, \tau)$ is a $T_{1 / 2}$-space, then the following conditions are equivalent

(1) $X$ is GO-connected;

(2) $X$ is TGO-connected;

(3) $X$ is connected.

(iii) A regular space $X$ is GO-connected if and only if $X$ is TGO-connected.

(iv) Let $f:(X, \tau) \rightarrow(Y, \sigma)$ be a surjection. Then

(a) If $f$ is $\theta$-generalized continuous and $X$ is TGO-connected, then $Y$ is connected.

(b) If $f$ is $\theta$-g-irresolute and $X$ is TGO-connected, then $Y$ is TGO-connected.

COROLLARY 6.3. If the product space $(X \times Y, \tau \times \sigma)$ is TGO-connected, then its factor space $(X, \tau)$ is TGO-connected.

THEOREM 6.4. Let $f:(X, \tau) \rightarrow(Y, \sigma)$ be $\theta$-g-continuous. Then the image of every $\theta$-closed, TGO-connected subset of $(X, \tau)$ is connected in $(Y, \sigma)$. 
Proof. Let $H$ be a $\theta$-closed and TGO-connected set in $(X, \tau)$. Then, by Proposition 5.9, the restriction of $f$ to $H, f \mid H:(H, \tau \mid H) \rightarrow(Y, \sigma)$, is $\theta$-g-continuous. For $f$, a function $r_{H}(f):(H, \tau \mid H) \rightarrow(f(H), \sigma \mid f(H))$ is well defined by $\left(r_{H}(f)\right)(x)=f(x)$ for any $x \in H$. Since $f \mid H=j \circ r_{H}(f)$, where $j:(f(H), \tau \mid f(H)) \rightarrow(Y, \sigma)$ is an inclusion. Then it is clear that $r_{H}(f)$ is $\theta$-g-continuous. In fact, for an open set $V$ of $(f(H), \sigma \mid f(H))$, take an open set $G \in \tau$ such that $G \cap f(H)=V$. Then $r_{H}(f)^{-1}(V)=$ $(f \mid H)^{-1}(G)$ is $\theta$-g-open. Now, by Observation 6.2(iv), $(f(H), \sigma \mid f(H))$ is connected and hence $f(H)$ is a connected subset of $(Y, \sigma)$.

ACKNOWLEDGEMENT. The authors thank the referee for his help in improving the quality of this paper.

Research supported partially by the Ella and Georg Ehrnrooth Foundation at Merita Bank, Finland and by the Japan-Scandinavia Sasakawa Foundation.

\section{REFERENCES}

[1] D. Andrijević, Semipreopen sets, Mat. Vesnik 38 (1986), no. 1, 24-32. MR 87j:54002. Zbl 604.54002.

[2] S. P. Arya and T. M. Nour, Characterizations of s-normal spaces, Indian J. Pure Appl. Math. 21 (1990), no. 8, 717-719. MR 91h:54004. Zbl 706.54021.

[3] K. Balachandran, P. Sundaram, and H. Maki, On generalized continuous maps in topological spaces, Mem. Fac. Sci. Kôchi Univ. Ser. A Math. 12 (1991), 5-13. MR 91m:54009. Zbl 736.54005 .

[4] P. Bhattacharyya and B. K. Lahiri, Semigeneralized closed sets in topology, Indian J. Math. 29 (1987), no. 3, 375-382. MR 90a:54004. Zbl 687.54002.

[5] A. S. Davis, Indexed systems of neighborhoods for general topological spaces, Amer. Math. Monthly 68 (1961), 886-893. MR 35\#4869. Zbl 106.15504.

[6] R. Devi, H. Maki, and K. Balachandran, Semi-generalized closed maps and generalized semi-closed maps, Mem. Fac. Sci. Kôchi Univ. Ser. A Math. 14 (1993), 41-54. MR 94b:54043. Zbl 787.54015.

[7] J. Dontchev, On generalizing semi-preopen sets, Mem. Fac. Sci. Kôchi Univ. Ser. A Math. 16 (1995), 35-48. MR 95k:54039. Zbl 833.54001.

[8] J. Dontchev and M. Ganster, On $\delta$-generalized closed sets and $T_{3 / 4}$-spaces, Mem. Fac. Sci. Kôchi Univ. Ser. A Math. 17 (1996), 15-31. MR 97d:54034. Zbl 853.54019.

[9] K. K. Dube, A note on $R_{0}$-topological spaces, Mat. Vesnik 11 (1974), no. 26, 203-208. MR 51 13982. Zbl 293.54014.

[10] W. Dunham, T T/2-spaces, Kyungpook Math. J. 17 (1977), no. 2, 161-169. MR 5710677. Zbl 382.54013 .

[11] T. Fukutake, On generalized closed sets in bitopological spaces, Bull. Fukuoka Univ. Ed. III 35 (1986), 19-28. MR 87i:54071. Zbl 624.54001.

[12] D. Janković, On some separation axioms and $\theta$-closure, Mat. Vesnik 32 (1980), no. 4, 439449. MR 84b:54039. Zbl 528.54016.

[13] E. Khalimsky, R. Kopperman, and P. R. Meyer, Computer graphics and connected topologies on finite ordered sets, Topology Appl. 36 (1990), no. 1, 1-17. MR 92c:54037. Zbl 709.54017.

[14] N. Levine, Semi-open sets and semi-continuity in topological spaces, Amer. Math. Monthly 70 (1963), 36-41. MR 29\#4025. Zbl 113.16304.

[15] _ Generalized closed sets in topology, Rend. Circ. Mat. Palermo (2) 19 (1970), 89-96. MR 46 4471. Zbl 231.54001.

[16] H. Maki, Generalized $\Lambda$-sets and the associated closure operator, The Special Issue in Commemoration of Prof. Kazusada IKEDA's Retirement (1986), 139-146. 
[17] H. Maki, R. Devi, and K. Balachandran, Generalized $\alpha$-closed sets in topology, Bull. Fukuoka Univ. Ed. III 42 (1993), 13-21. Zbl 888.54005.

[18] _ Associated topologies of generalized $\alpha$-closed sets and $\alpha$-generalized closed sets, Mem. Fac. Sci. Kôchi Univ. Ser. A Math. 15 (1994), 51-63. MR 95c:54037. Zbl 821.54002.

[19] A. S. Mashhour, M. E. Abd El Monsef, and S. N. El Deep, On precontinuous and weak precontinuous mappings, Proc. Math. Phys. Soc. Egypt (1982), no. 53, 47-53. MR 87c:54002. Zbl 571.54011.

[20] O. Njåstad, On some classes of nearly open sets, Pacific J. Math. 15 (1965), 961-970. MR 33\#3245. Zbl 137.41903.

[21] T. Noiri, On $\delta$-continuous functions, J. Korean Math. Soc. 16 (1979;1980), no. 2, 161-166. MR 82b:54020. Zbl 435.54010.

[22] H. Ogata, Operations on topological spaces and associated topology, Math. Japon. 36 (1991), no. 1, 175-184. MR 92c:54004. Zbl 725.54004.

[23] N. Palaniappan and K. C. Rao, Regular generalized closed sets, Kyungpook Math. J. 33 (1993), no. 2, 211-219. MR 94k:54018. Zbl 794.54002.

[24] P. Sundaram, H. Maki, and K. Balachandran, Semi-generalized continuous maps and semi-T $T_{1 / 2}$ spaces, Bull. Fukuoka Univ. Ed. III 40 (1991), 33-40. MR 92j:54032c. Zbl 790.54010 .

[25] J. Umehara, H. Maki, and T. Noiri, Bioperations on topological spaces and some separation axioms, Mem. Fac. Sci. Kôchi Univ. Ser. A Math. 13 (1992), 45-59. MR 93b:54020. Zbl 751.54010 .

[26] N. V. Veličko, H-closed topological spaces, Amer. Math. Soc. Transl. 78 (1968), 103-118. Zbl 183.27302.

[27] A. Wilansky, Between $T_{1}$ and $T_{2}$, Amer. Math. Monthly 74 (1967), 261-266. MR 34\#8367. Zbl 147.22704.

DONTCHEV: DePARTMENT OF MATHEMATICS, PL 4, YliopistonKATU 5, UNIVERSITY OF HELSINKI, 00014 HELSINKI 10, FINLAND

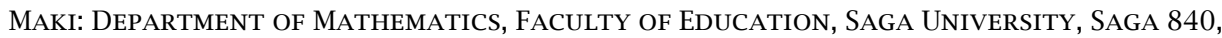
JAPAN 


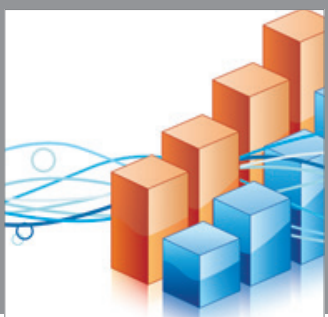

Advances in

Operations Research

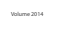

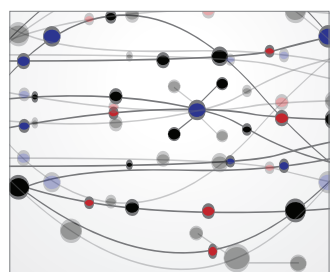

\section{The Scientific} World Journal
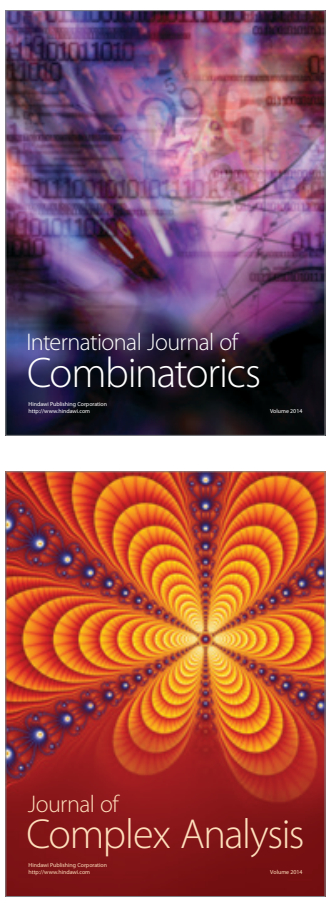

International Journal of

Mathematics and

Mathematical

Sciences
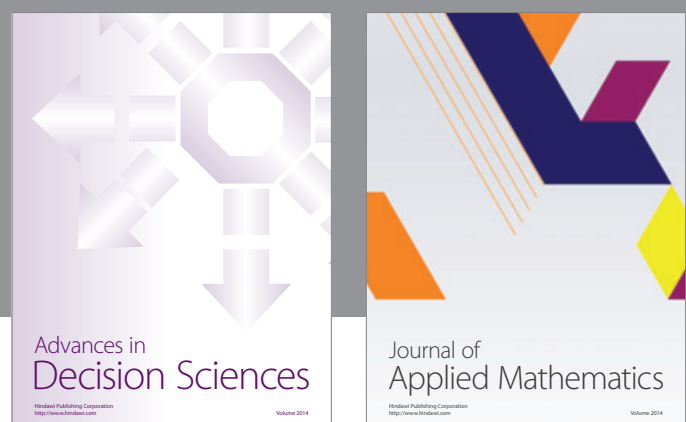

Journal of

Applied Mathematics
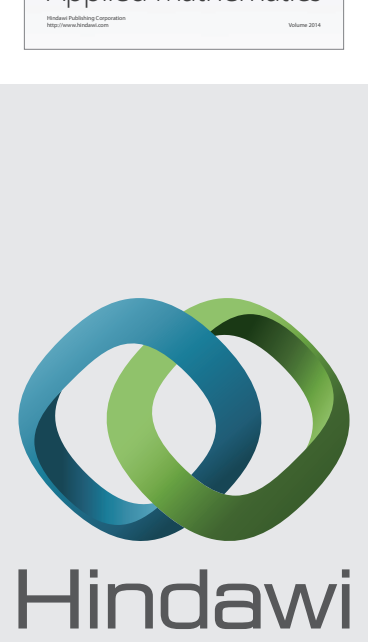

Submit your manuscripts at http://www.hindawi.com
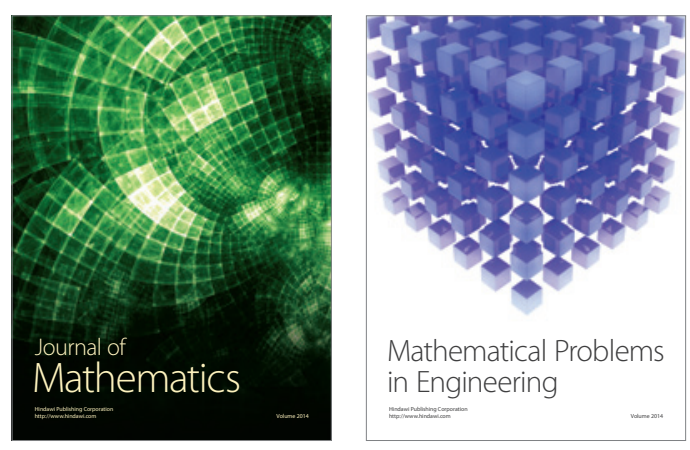

Mathematical Problems in Engineering
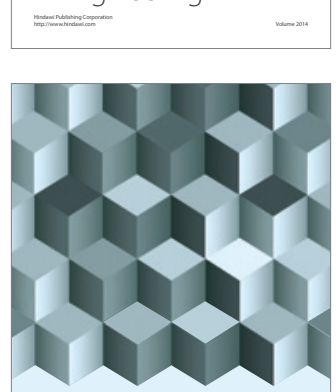

Journal of

Function Spaces
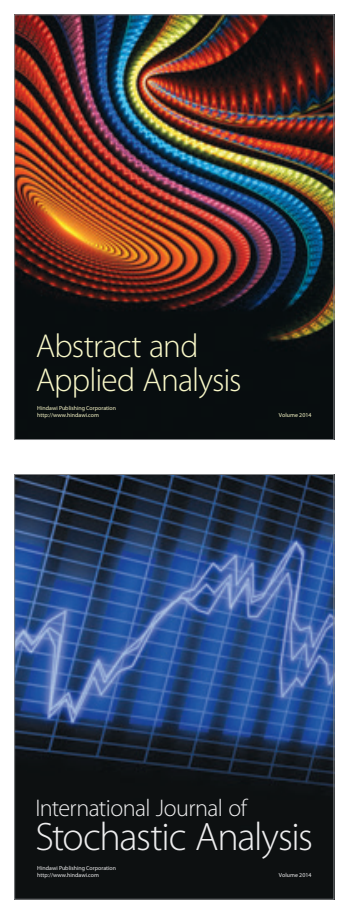

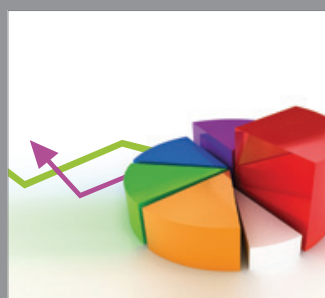

ournal of

Probability and Statistics

Promensencen
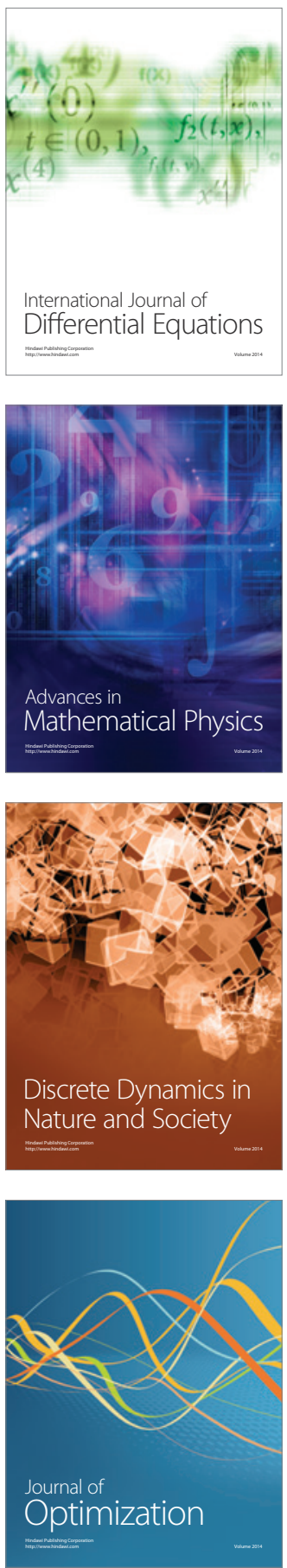